\title{
Article \\ Postpubertal Effects of the Rapid Maxillary Expansion and Facial Mask versus the Removable Mandibular Retractor for the Early Treatment of Class III Malocclusion: A Study on Lateral Cephalograms
}

\author{
Veronica Giuntini ${ }^{1}$, Matteo Camporesi ${ }^{2}$, Valeria Barone ${ }^{3,4} \mathbb{D}^{\mathbb{D}}$, Matilde Marino Merlo ${ }^{1}$, Cosimo Nardi $^{5} \mathbb{( D}$, \\ Debora Franceschi ${ }^{1}$ (D) and Lorenzo Franchi ${ }^{1, *(D)}$ \\ 1 Department of Experimental and Clinical Medicine, University of Florence, 50121 Florence, Italy; \\ veronica.giuntini@unifi.it (V.G.); matilde.marinomerlo@unifi.it (M.M.M.); debora.franceschi@unifi.it (D.F.) \\ 2 Azienda Ospedaliero-Universitaria Careggi, 50134 Florence, Italy; matteo_camporesi@yahoo.it \\ 3 Private Practice, 50100 Florence, Italy; valeriabarone@hotmail.it \\ 4 Private Practice, 59100 Prato, Italy \\ 5 Department of Experimental and Clinical Biomedical Sciences, Radiodiagnostic Unit n. 2, \\ University of Florence, 50134 Florence, Italy; cosimo.nardi@unifi.it \\ * Correspondence: lorenzo.franchi@unifi.it; Tel.: +39-055-794-5602
}

Citation: Giuntini, V.; Camporesi, M.; Barone, V.; Marino Merlo, M.; Nardi,

C.; Franceschi, D.; Franchi, L.

Postpubertal Effects of the Rapid

Maxillary Expansion and Facial Mask

versus the Removable Mandibular

Retractor for the Early Treatment of

Class III Malocclusion: A Study on

Lateral Cephalograms. Appl. Sci.

2021, 11, 8393. https://doi.org/

10.3390/app11188393

Academic Editor: Gianrico Spagnuolo

Received: 13 August 2021

Accepted: 5 September 2021

Published: 10 September 2021

Publisher's Note: MDPI stays neutral with regard to jurisdictional claims in published maps and institutional affiliations.

Copyright: (C) 2021 by the authors. Licensee MDPI, Basel, Switzerland. This article is an open access article distributed under the terms and conditions of the Creative Commons Attribution (CC BY) license (https:/ / creativecommons.org/licenses/by/ $4.0 /)$.
Abstract: The aim of this study was to compare the efficacy of early treatment of Class III malocclusions with rapid maxillary expansion (RME) and facial mask (FM) versus the removable mandibular retractor (RMR) re-evaluated at a postpubertal observation on lateral cephalograms. All prepubertal patients with Class III malocclusion treated consecutively from 1986 to 2013 by means of RME/FM or RMR were analyzed. Twenty-nine patients treated with RME/FM therapy and 23 patients treated with RMR were selected. Lateral cephalograms were available at 3 time points, before treatment (T1), at the end of active treatment (T2), and at a postpubertal observation (T3). Statistical comparisons were performed with independent sample $t$ tests or Mann-Whitney tests. During the T1-T3 interval, a significantly greater maxillary protraction (SNA $+1.5 \mathrm{~mm}, p=0.031$ ) and significantly greater improvements in ANB and Wits appraisal ( +1.9 degrees, $p=0.002$, and $+2.2 \mathrm{~mm}, p=0.012$, respectively) were recorded in the RME/FM group. No statistically significant changes could be found in vertical skeletal measurements. In the dentoalveolar region, the RME/FM group showed a significantly greater correction of the molar relationship $(-1.5 \mathrm{~mm}, p=0.021)$. Early treatment of Class III malocclusion with RME/FM protocol in comparison with RMR protocol showed a greater maxillary advancement and greater improvements in sagittal skeletal Class III relationships.

Keywords: Class III malocclusion; lateral cephalogram; cephalometrics

\section{Introduction}

Treatment of Class III malocclusion is one of the greatest challenges in contemporary orthodontics [1,2]. Different treatment approaches have been suggested for the early treatment of Class III malocclusion such as the facial mask [3,4], Frankel III Appliance [5], the Class III Bionator [6], the chin cup [7], and the removable mandibular retractor (RMR) [2]. Rapid maxillary expansion and facial mask (RME/FM) therapy is the most common orthopedic treatment protocol for Class III malocclusion [3,4]. RMR has been proposed as a simple functional appliance in the treatment of Class III patients [8-14].

Some studies showed the ability of the RMR of changing mandibular growth pattern in the early treatment of Class III deformities either in the early or late mixed dentitions [11,13]. Saleh et al. [13] in a randomized clinical trial reported that skeletal changes in mandibular shape in the treated group could be described as an "anterior morphogenetic rotation of the mandible" according to Lavergne and Gasson [15]. This RCT [13], however, analyzed 
a follow-up period of 14.5 months. In the literature no data are available regarding the outcomes of RMR therapy re-evaluated at a postpubertal observation. This information is essential for the evaluation of orthopedic treatment of patients with a Class III malocclusion, as a significant tendency to restore Class III dentoskeletal relationships after maxillary protraction has been demonstrated, especially after the pubertal growth peak [4]. Second, pubertal growth tends to last longer in Class III subjects compared with Class I subjects [16].

On the other end, postpubertal assessments of the treatment effects produced by different orthopedic and orthodontic approaches are available. Mousoulea et al. [7] reported that chin-cup therapy can induce favorable short-term changes with controversy about the long-term stability. As for RME/FM, Lin et al. [4] showed that the posttreatment changes in most variables reflected significant relapse, including backward retrusion of the maxilla. Moreover, no previous study has compared the dento-skeletal effects produced by the RMR versus RME/FM.

The aim of this study, therefore, was to compare postpubertal treatment effects produced by early treatment of Class III malocclusion with RME/FM versus RMR evaluated on lateral cephalograms.

\section{Materials and Methods}

This study was approved by the Pediatric Ethics Committee of the Region of Tuscany $(241 / 2019)$, and informed consent was obtained from the subjects' parents. For this retrospective study all the patients with Class III malocclusion treated consecutively from 1986 to 2013 by means of RME/FM or RMR at the Orthodontic Clinic of the Careggi University Hospital in Florence, Italy, were analyzed.

The sample inclusion criteria were:

- $\quad$ European ancestry (Caucasian ethnicity);

- A negative Wits appraisal greater than $-2.0 \mathrm{~mm}$;

- Anterior crossbite or edge-edge incisor relationship;

- Class III molar relationship;

- No discrepancy between centric occlusion and centric relation (indicating pseudoClass III malocclusion);

- $\quad$ Prepuberal skeletal maturation (CS1-CS3) [17];

- $\quad$ No congenitally missing teeth;

- No craniofacial syndromes.

Moreover, 3 lateral cephalograms had to be available for each patient:

- Pre-treatment lateral cephalogram before therapy (T1);

- $\quad$ Lateral cephalogram at the end of the active treatment phase (T2);

- Lateral cephalogram at a postpubertal stage of skeletal maturation (CS4-6) (T3).

Lateral cephalograms were scanned with the same resolution (150 dpi) and then digitized by one investigator (V.B.). A customized digitization regimen (Viewbox, version 4.0, dHAL Software, Kifissia, Greece) was created and used for cephalometric evaluation. Ten variables (three linear and seven angular) were generated for each tracing (Figure 1). Magnification factor was standardized to life size $(0 \%)$.

\subsection{RME/FM Protocol}

RME/FM protocol consisted of 3 components: a Hyrax maxillary expansion appliance, a facemask, and heavy elastics [18] (Figure 2). Treatment began with the insertion of a bonded or banded Hyrax maxillary expander to which vestibular hooks that extended to the maxillary deciduous canine were attached. Patients' parents were instructed to activate the expander once or twice a day until the desired transverse width was obtained. Immediately after expansion, patients received the facemask. Elastics were stretched from the soldered hooks of the expander to the support bar of the facemask and were inclined downward and forward (approximately 30 degrees to the occlusal plane), creating orthopedic forces of 400 to $500 \mathrm{~g}$ per side. Patients were instructed to wear the facemask for at least $14 \mathrm{~h}$ per day. 
All patients were treated to at least a positive overjet before discontinuing treatment; most patients were overcorrected to-ward Class II occlusal relationships. The mean duration of RME/FM protocol was $1.1 \pm 0.2$ years. $52 \%$ of patients received fixed appliance therapy again between $\mathrm{T} 2$ and $\mathrm{T} 3$.

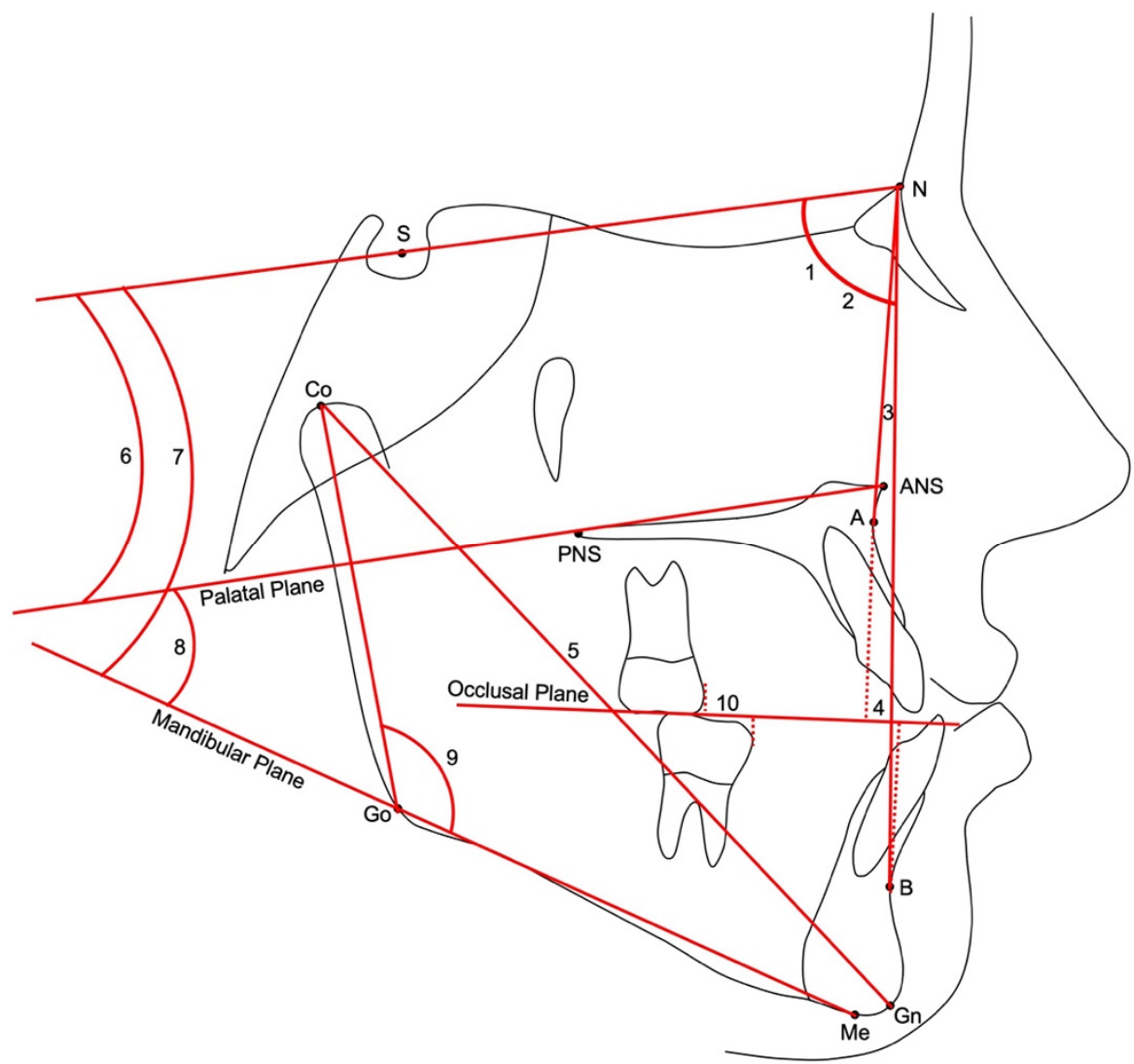

Figure 1. Cephalometric landmarks and measurements. Cephalometric landmarks. S: Sella; N: Nasion; ANS: Anterior Nasal Spine; PNS: Posterior Nasal Spine; A: A point; B: B point; Gn: Gnathion; Me: Menton; Go: Gonion; Co: Condylion. Cephalometric angular measurements: (1) SNA; (2) SNB; (3) ANB; (6) SN to Palatal Plane; (7) SN to Mandibular Plane; (8) Palatal Plane to Mandibular Plane; (9) CoGoMe Mandibular Angle. Cephalometric linear measurements: (4) Wits appraisal; (5) CoGn total mandibular length; (10) Molar relationship.

\subsection{RMR Protocol}

RMR was described by Tollaro et al. [8,9] (Figure 3). The appliance consists of an upper resin plate attached to the maxillary arch with Adams' clasps, supporting a labial arch that extends to the cervical margin of the lower incisors. The labial arch is activated to be $2 \mathrm{~mm}$ in front of the mandibular incisors when the mandible is forced to maximum retrusion. The archwire is thus intended to act as a stop to the sagittal movement of the mandible. The children were instructed to wear the appliance for at least $14 \mathrm{~h}$ a day until the first sign of a corrected anterior crossbite. Thereafter, patients wore the appliance only at night until the end of the observation period. $35 \%$ of the patients received fixed appliance therapy between $\mathrm{T} 2$ and $\mathrm{T} 3$. 

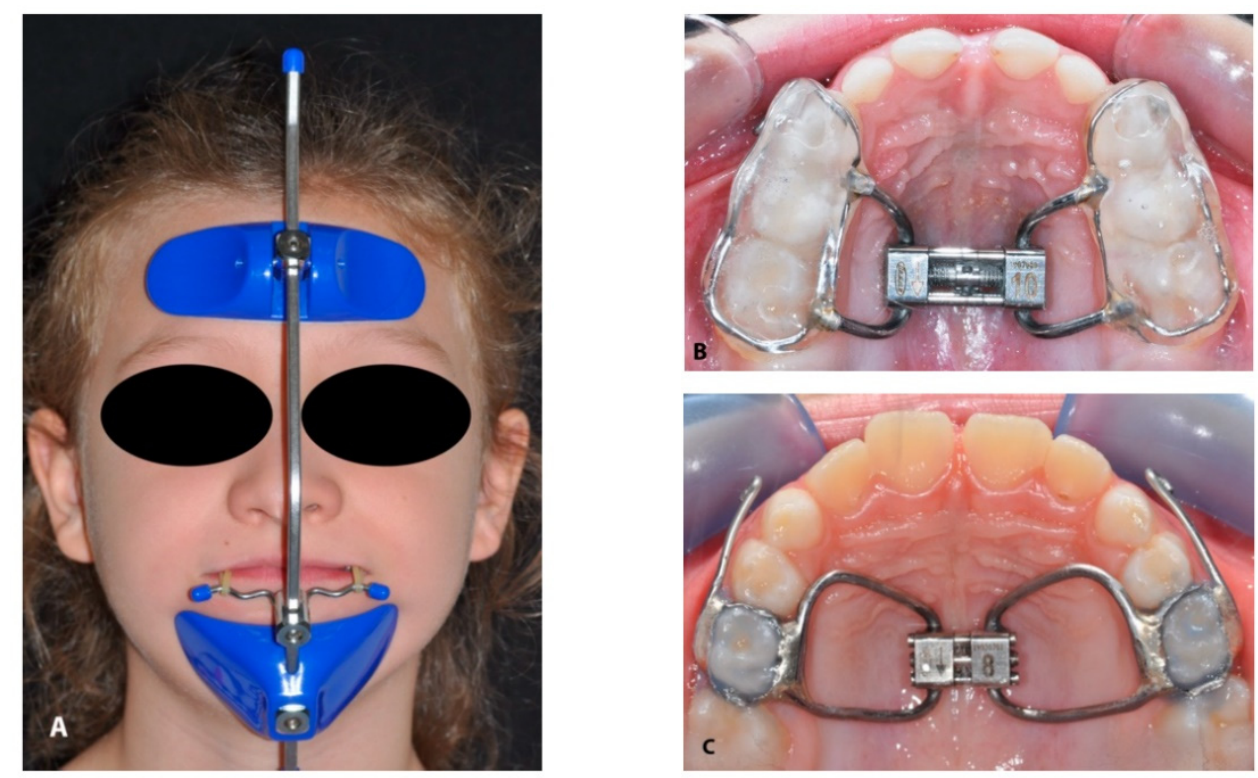

Figure 2. Facemask (A) and bonded (B) and banded (C) Hyrax maxillary expansion appliances.

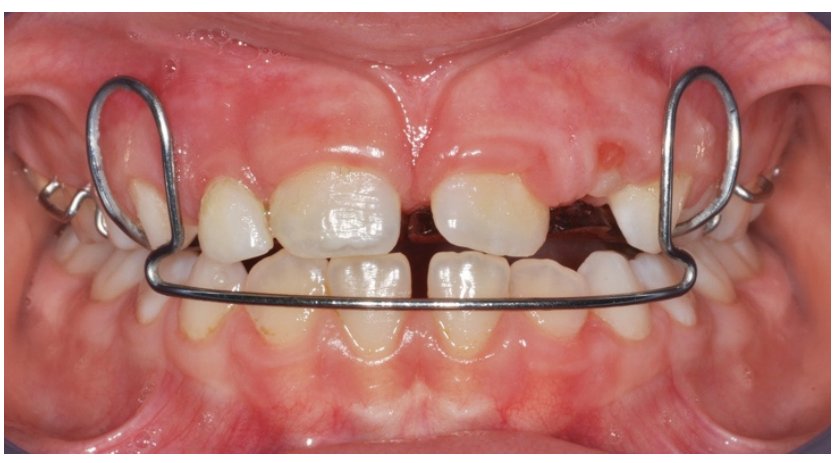

Figure 3. Removable Mandibular Retractor.

\subsection{Method Error}

In order to evaluate the intra-operator method error, 20 lateral cephalograms randomly selected from the two groups were re-digitized after 10 days by the same operator (V.B.). The error was calculated by means of method of moments estimator (MME) [19].

\subsection{Statistical Analysis}

The primary aim of the study was to compare the postpubertal dentoskeletal effects of early Class III treatment with RME/FM versus RMR followed by fixed appliances. Therefore, statistical comparisons were performed for the craniofacial features at baseline (T1) and for the T1-T3 changes with independent sample $t$ tests. When data were not normally distributed, Mann-Whitney tests were used.

As secondary statistical analysis, between-group comparisons for the T1-T2 and T2T3 changes were carried out. All statistical computations were performed with a statistical software (SPSS Statistics for Mac, Version 26.0, IBM Corp. Armonk, NY, USA).

\section{Results}

The method error of cephalometric measurements ranged between 0.5 (molar relationship) and 1.1 (Wits appraisal) $\mathrm{mm}$ for linear measurements and between $0.5^{\circ}$ (SNB) and $1.1^{\circ}$ (CoGoMe) for angular measurements.

Fifty-two patients treated consecutively with either RME/FM therapy (RME/FM group, 29 patients, 13 females and 16 males) or RMR therapy (RMR group, 23 patients, 
13 females and 10 males) at the Orthodontic Clinic of the Careggi University Hospital in Florence, Italy, were analyzed. Mean ages for the RME/FM group were $7.4 \pm 1.6$ years at $\mathrm{T} 1,9.0 \pm 1.7$ years at T2, and $15.1 \pm 1.4$ years at T3. Mean ages for the RMR group were $7.7 \pm 2.5$ years at $\mathrm{T} 1,9.4 \pm 2.3$ years at $\mathrm{T} 2$, and $14.8 \pm 1.3$ years at $\mathrm{T} 3$.

No statistically significant differences between the two groups were found for any of the cephalometric variables at baseline (Table 1) with the exception of a significantly greater retrusion of the maxilla in the RME/FM group than the RMR group (SNA 78.2 and 80.4 degrees, respectively).

Table 1. Descriptive statistics and statistical comparisons (independent sample $t$ test) for the starting forms (T1).

\begin{tabular}{ccccccccc}
\hline \multirow{2}{*}{ Variables } & \multicolumn{2}{c}{ RME/FM } & \multicolumn{2}{c}{ RMR } & & \multirow{2}{c}{ Diff. } & $p$ & \multicolumn{2}{c}{ 95\% C.I. } \\
\cline { 2 - 4 } & Mean & SD & Mean & SD & & & Lower & Upper \\
\hline Age (years) & 7.4 & 1.6 & 7.7 & 2.6 & -0.3 & 0.705 & -1.4 & 1.0 \\
SNA (deg) & 78.2 & 3.4 & 80.4 & 3.1 & -2.2 & 0.022 & -4.0 & -0.3 \\
SNB (deg) & 77.1 & 3.4 & 78.7 & 3.4 & -1.6 & 0.096 & -3.5 & 0.3 \\
ANB (deg) & 1.1 & 1.7 & 1.7 & 2.7 & -0.6 & 0.359 & -1.8 & 0.7 \\
Wits (mm) & -4.8 & 2.6 & -3.8 & 3.1 & -1.0 & 0.216 & -2.6 & 0.6 \\
CoGn (mm) & 96.0 & 6.5 & 96.8 & 6.8 & -0.8 & 0.678 & -4.5 & 2.9 \\
SN-Pal. Pl. (deg) & 9.1 & 3.6 & 7.8 & 3.4 & 1.3 & 0.210 & -0.7 & 3.2 \\
SN-Mand. Pl. (deg) & 38.2 & 4.8 & 35.4 & 5.1 & 2.8 & 0.053 & 0.0 & 5.5 \\
Pal. Pl.-Mand. Pl. (deg) & 29.1 & 4.4 & 27.6 & 4.8 & 1.5 & 0.251 & -1.1 & 4.0 \\
CoGoMe (deg) & 128.7 & 4.7 & 127.4 & 4.4 & 1.3 & 0.326 & -1.3 & 3.8 \\
Mol. Rel. (mm) & 2.4 & 2.0 & 1.7 & 1.8 & 0.7 & 0.188 & -0.4 & 1.8 \\
\hline
\end{tabular}

deg.: degrees; SD: Standard Deviation; Pal.: Palatal; Pl.: Plane; Mand.: Mandibular; Mol.: Molar; Rel.: Relationship Diff.: Difference; C.I.: Confidence Interval.

As for the T1-T3 changes (Table 2), a significantly greater maxillary protraction $(\mathrm{SNA}+1.5 \mathrm{~mm}$ ) was recorded in the RME/FM group with respect to the RMR group. The RME/FM group also showed significantly greater improvements in ANB and Wits appraisal ( +1.9 degrees and $+2.2 \mathrm{~mm}$, respectively) with the RMR group presenting a slight worsening of these measurements ( -0.9 degrees and $-0.7 \mathrm{~mm}$, respectively). No statistically significant changes could be found in vertical skeletal measurements. In the dentoalveolar region, the RME/FM group showed a significantly greater correction of the molar relationship $(-1.5 \mathrm{~mm})$ with the RMR group presenting a worsening of the Class III molar relationship (+1.2 $\mathrm{mm})$.

Table 2. Descriptive statistics and statistical comparisons (independent sample $t$ test or MannWhitney U test) for the T1-T3 changes.

\begin{tabular}{|c|c|c|c|c|c|c|c|c|}
\hline \multirow[b]{2}{*}{ Variables } & \multicolumn{2}{|c|}{ RME/FM } & \multicolumn{2}{|c|}{ RMR } & \multirow[b]{2}{*}{ Diff. } & \multirow[b]{2}{*}{$p$} & \multicolumn{2}{|c|}{ 95\% C.I. } \\
\hline & $\begin{array}{c}\text { Mean } \\
\text { Median }\end{array}$ & $\begin{array}{c}\mathrm{SD} \\
I Q R\end{array}$ & $\begin{array}{l}\text { Mean } \\
\text { Median }\end{array}$ & $\begin{array}{c}\mathrm{SD} \\
I Q R\end{array}$ & & & Lower & Upper \\
\hline Age (years) & 7.6 & 2.3 & 7.1 & 2.8 & 0.5 & 0.473 & -0.9 & 1.9 \\
\hline SNA (deg) & 1.9 & 2.0 & 0.4 & 2.5 & 1.5 & 0.031 & 0.1 & 2.7 \\
\hline SNB (deg) & 1.0 & 2.6 & 1.3 & 1.8 & -0.3 & 0.597 & -1.6 & 0.9 \\
\hline ANB (deg) & 1.0 & 2.0 & -0.9 & 2.0 & 1.9 & 0.002 & 0.8 & 3.0 \\
\hline Wits (mm) & 1.5 & 3.0 & -0.7 & 3.1 & 2.2 & 0.012 & 0.5 & 3.9 \\
\hline CoGn $(\mathrm{mm})$ & 18.3 & 7.1 & 17.4 & 7.0 & 0.9 & 0.649 & -3.1 & 4.9 \\
\hline SN-Pal. Pl. (deg) & 0.2 & 2.3 & 0.3 & 2.7 & -0.1 & 0.918 & -1.4 & 1.3 \\
\hline SN-Mand. Pl. (deg) & -1.7 & 3.2 & -2.8 & 3.5 & 1.1 & 0.232 & -0.7 & 3.0 \\
\hline Pal. Pl.-Mand. Pl. (deg) & -1.9 & 2.8 & -3.1 & 4.2 & 1.2 & 0.224 & -0.8 & 3.2 \\
\hline CoGoMe (deg) & -3.2 & 3.2 & -3.6 & 4.6 & 0.4 & 0.978 & & \\
\hline Mol. Rel. (mm) & -0.3 & 2.7 & 1.2 & 1.6 & -1.5 & 0.021 & -2.8 & -0.2 \\
\hline
\end{tabular}

deg.: degrees; SD: Standard Deviation; Pal.: Palatal; Pl.: Plane; Mand.: Mandibular; Mol.: Molar; Rel.: Relationship; Diff.: Difference; C.I.: Confidence Interval; IQR: Interquartile Range. 
In the short-term (T1-T2, Table 3) the RME/FM group showed a significantly greater maxillary protraction (SNA +2.3 degrees), a significantly greater control in mandibular sagittal position (SNB -1.3 degrees), and significantly greater improvements in both ANB and Wits appraisal (+3.6 degrees and $+2.6 \mathrm{~mm}$, respectively) with respect to the RMR group. As for the vertical skeletal relationships, the RME/FM group presented with significantly greater increases in both facial divergence (SN to Mandibular Plane) and intermaxillary divergence (Palatal plane to mandibular Plane) $(+1.8$ and +2.5 degrees, respectively). In the RME/FM group a significantly greater correction of the molar relationship $(-2.5 \mathrm{~mm})$ occurred with respect to the RMR group.

Table 3. Descriptive statistics and statistical comparisons (independent sample $t$ test) for the T1T2 changes.

\begin{tabular}{|c|c|c|c|c|c|c|c|c|}
\hline \multirow{2}{*}{ Variables } & \multicolumn{2}{|c|}{ RME/FM } & \multicolumn{2}{|c|}{ RMR } & \multirow{2}{*}{ Diff. } & \multirow{2}{*}{$p$} & \multicolumn{2}{|c|}{ 95\% C.I. } \\
\hline & Mean & SD & Mean & SD & & & Lower & Upper \\
\hline Age (years) & 1.6 & 0.4 & 1.8 & 1.1 & -0.2 & 0.425 & -0.6 & 0.3 \\
\hline SNA (deg) & 1.9 & 1.3 & -0.4 & 2.2 & 2.3 & 0.000 & 1.2 & 3.2 \\
\hline SNB (deg) & -1.3 & 1.5 & 0.0 & 1.6 & -1.3 & 0.006 & -2.1 & -0.4 \\
\hline ANB (deg) & 3.2 & 1.8 & -0.4 & 1.7 & 3.6 & 0.000 & 2.6 & 4.6 \\
\hline Wits (mm) & 1.6 & 2.7 & -1.0 & 2.7 & 2.6 & 0.001 & 1.1 & 4.1 \\
\hline CoGn $(\mathrm{mm})$ & 4.1 & 2.5 & 4.6 & 2.6 & -0.5 & 0.483 & -1.9 & 0.9 \\
\hline SN-Pal. Pl. (deg) & -0.7 & 2.1 & 0.0 & 2.3 & -0.7 & 0.229 & -2.0 & 0.5 \\
\hline SN-Mand. Pl. (deg) & 1.5 & 2.0 & -0.3 & 2.5 & 1.8 & 0.007 & 0.5 & 3.0 \\
\hline Pal. Pl.-Mand. Pl. (deg) & 2.2 & 3.2 & -0.3 & 2.1 & 2.5 & 0.002 & 1.0 & 4.1 \\
\hline CoGoMe (deg) & -1.3 & 2.0 & -1.0 & 2.9 & -0.3 & 0.626 & -1.7 & 1.0 \\
\hline Mol. Rel. (mm) & -2.4 & 2.5 & 0.1 & 1.5 & -2.5 & 0.000 & -3.7 & -1.3 \\
\hline
\end{tabular}

deg.: degrees; SD: Standard Deviation; Pal.: Palatal; Pl.: Plane; Mand.: Mandibular; Mol.: Molar; Rel.: Relationship Diff.: Difference; C.I.: Confidence Interval.

No significant differences were found between the two groups in the post-treatment changes (T2-T3, Table 4) except for the ANB that showed a significantly greater worsening in the RME/FM group ( -1.7 degrees).

Table 4. Descriptive statistics and statistical comparisons (independent sample $t$ test) for the T2T3 changes.

\begin{tabular}{|c|c|c|c|c|c|c|c|c|}
\hline \multirow[b]{2}{*}{ Variables } & \multicolumn{2}{|c|}{ RME/FM } & \multicolumn{2}{|c|}{ RMR } & \multirow[b]{2}{*}{ Diff. } & \multirow[b]{2}{*}{$p$} & \multicolumn{2}{|c|}{ 95\% C.I. } \\
\hline & $\begin{array}{c}\text { Mean } \\
\text { Median }\end{array}$ & $\begin{array}{c}\text { SD } \\
I Q R\end{array}$ & $\begin{array}{l}\text { Mean } \\
\text { Median }\end{array}$ & $\begin{array}{c}\text { SD } \\
I Q R\end{array}$ & & & Lower & Upper \\
\hline Age (years) & 6.0 & 2.3 & 5.3 & 2.5 & 0.7 & 0.305 & -0.6 & 2.0 \\
\hline SNA (deg) & 0.0 & 1.9 & 0.8 & 1.9 & -0.8 & 0.138 & -1.9 & 0.3 \\
\hline SNB (deg) & 2.2 & 2.5 & 1.3 & 1.5 & 0.9 & 0.128 & -0.3 & 2.1 \\
\hline ANB (deg) & -2.2 & 2.2 & -0.5 & 1.5 & -1.7 & 0.002 & -2.8 & -0.6 \\
\hline Wits (mm) & -0.1 & 3.2 & 0.3 & 1.9 & -0.4 & 0.596 & -1.9 & 1.1 \\
\hline CoGn $(\mathrm{mm})$ & 14.3 & 6.3 & 12.8 & 6.9 & 1.5 & 0.446 & -2.3 & 5.1 \\
\hline SN-Pal. Pl. (deg) & 1.0 & 2.1 & 0.3 & 2.2 & 0.7 & 0.266 & -0.5 & 1.9 \\
\hline SN-Mand. Pl. (deg) & -3.2 & 3.5 & -2.5 & 2.7 & -0.7 & 0.479 & -2.4 & 1.2 \\
\hline Pal. Pl.-Mand. Pl. (deg) & -4.1 & 3.5 & -2.8 & 3.6 & -1.3 & 0.178 & -3.3 & 0.6 \\
\hline CoGoMe (deg) & -2.2 & 3.0 & -2.9 & 3.4 & 0.7 & 0.425 & -1.1 & 2.5 \\
\hline Mol. Rel. (mm) & 1.7 & 3.5 & 1.3 & 2.4 & 0.7 & 0.320 & & \\
\hline
\end{tabular}

deg.: degrees; SD: Standard Deviation; Pal.: Palatal; Pl.: Plane; Mand.: Mandibular; Mol.: Molar; Rel.. Relationship Diff.: Difference; C.I.: Confidence Interval; IQR: Interquartile Range.

\section{Discussion}

Class III malocclusion in growing children is one of the most challenging malocclusions to treat. The most common treatment protocols for Class III malocclusion include RME/FM therapy, function regulator, chin cup, and other approaches $[1,2,5,7,20,21]$. 
RME/FM therapy is the most common orthopedic treatment protocol for Class III malocclusion [1-4]. Many functional appliances have been proposed to correct Class III deformities in the early developmental phases, and one of these functional appliances is the RMR [8-14]. Although the short-term effects produced by RME/FM and RMR have been described previously [1-4], limited information on the medium- (postpubertal) to long-term (end of active growth) stability is available [4]. There is lack of information with regard to the comparison of the dento-skeletal effects produced by RME/FM and RMR, re-evaluated at a postpubertal stage of skeletal maturation.

The results of the T1-T2 interval (Table 3) can be regarded as the short-term outcomes comparison of the two analyzed protocols. Significant improvements in intermaxillary sagittal skeletal relationships (ANB and Wits appraisal +3.6 degrees and $+2.6 \mathrm{~mm}$, respectively) were recorded in the RME/FM treatment group during the T1-T2 interval in comparison with the RMR appliance. In particular, RME/FM produced significantly greater maxillary protraction (SNA +2.3 degrees) and control of the sagittal mandibular position (SNB -1.3 degrees) (Table 3 ). These favorable intermaxillary sagittal changes were similar to those reported in previous studies [1-4]. RME/FM therapy produced a significantly greater clockwise mandibular rotation (SN-Mand. P1. +1.8 degrees) associated with significantly greater increases in intermaxillary divergence (Pal. Pl--Mand. Pl. +2.5 degrees) with respect to RMR. This result is in agreement with previous studies [1-4]. As for the short-term dental changes, the RME/FM compared with RMR showed a significantly greater correction of molar relationship $(-2.5 \mathrm{~mm})$ (Table 3).

The comparison of the outcomes during the post-treatment T2-T3 interval, including the pubertal growth spurt, showed a significantly greater relapse in intermaxillary sagittal relationships (ANB $-1.7 \mathrm{~mm}$ ) in the RME/FM group (Table 4). This relapse was due mainly to unfavorable mandibular sagittal changes rather than maxillary sagittal changes. Additionally, the greater relapse in intermaxillary sagittal relationships could be related probably to a greater post-treatment rebound of the mandibular clockwise rotation in the $\mathrm{RME} / \mathrm{FM}$ group. Our results agree with previous findings that Class III craniofacial growth pattern tends to be re-established after active orthopedic treatment with RME/FM [4]. As for the vertical skeletal relationships, both groups showed a counterclockwise rotation of the mandible that was associated with a reduction in intermaxillary divergence (Table 4). This finding is also in agreement with previous reports on RME/FM [4].

The analysis of the outcomes during the T1-T3 interval can be considered the mediumterm results for both treatment protocols (Table 2). The comparison of the results showed that the greater maxillary advancement was performed by the RME/FM protocol in comparison with the RMR protocol (SNA +1.5 degrees). This result agrees with previous studies [22,23] that showed respectively a maxillary advancement of $1.2 \mathrm{~mm}$ and $1.4 \mathrm{~mm}$ of Point A to Nasion perpendicular in Class III patients treated by RME/FM with respect to subjects with untreated Class III malocclusion when evaluated at a postpubertal observation. No significant differences were found between the two protocols in the correction of mandibular protrusion. The RME/FM protocol showed greater efficacy than RMR protocol in the correction of skeletal sagittal Class III relationship (ANB and Wits appraisal +1.9 degrees and $+2.2 \mathrm{~mm}$, respectively). Both protocols exhibited a counterclockwise rotation of the mandible that was associated with a decrease in intermaxillary divergence (Table 2). This result for the RME/FM protocol is in agreement with previous investigations [22,23]. As for the dental changes the RME/FM protocol produced a significantly greater correction of the molar relationship $(-1.5 \mathrm{~mm})$ with respect to RMR that actually induced a worsening of the Class III molar relationship (+1.2 mm).

In the RME/FM group 52\% received phase 2 treatment before facial growth end be-cause there was lack of space in the upper arch for the eruption of the maxillary permanent canines or because it was decided, in agreement with patients' parents, to re-fine the occlusion.

A limitation of the present study was its retrospective nature that could increase the risk for selection bias. We tried to control this bias by including in both groups all consecu- 
tively treated patients who presented with the inclusion criteria. Another limitation was the absence of a control sample of subjects with untreated Class III malocclusion. However, it is very difficult to find a contemporary sample with untreated Class III malocclusion mainly for ethical reasons. Finally, the last observation of the present study was taken at a postpubertal phase of skeletal maturation while, ideally, the final follow-up should have been collected at the end of active growth.

The results of the present study clearly demonstrated that if the aim is to produce a favorable correction of Class III skeletal imbalance, the RME/FM protocol should be preferred with respect to RMR. RMR can be regarded as a useful protocol in the treatment of pseudo-Class III malocclusion with mandibular functional anterior shift or as a retention appliance after RME/FM.

\section{Conclusions}

RME/FM can produce favorable sagittal skeletal changes in both the short and medium term. In the medium term, early treatment of Class III malocclusion with RME/FM protocol in comparison with RMR protocol shows a greater maxillary advancement and greater improvements in sagittal skeletal Class III relationships.

Author Contributions: Conceptualization, V.G. and L.F.; Methodology, V.G. and L.F; Formal Analysis, M.C., V.B., and M.M.M.; Investigation, V.G., L.F., M.C., V.B., and M.M.M.; Resources, V.B. and M.M.M.; Data Curation, V.G. and L.F.; Writing_-Original Draft Preparation, L.F. and M.C.; WritingReview and Editing, L.F.; Visualization, V.G., M.C., D.F., and C.N.; Supervision, V.G., M.C., D.F., and C.N.; Project Administration, V.G. and L.F. All authors have read and agreed to the published version of the manuscript.

Funding: This research received no external funding.

Institutional Review Board Statement: The study was conducted according to the guidelines of the Declaration of Helsinki and approved by the Pediatric Ethics Committee of the Region of Tuscany, Italy (protocol code 241/2019 approved on 20 December 2019).

Informed Consent Statement: Informed consent was obtained from all subjects involved in the study.

Data Availability Statement: The data will be available on request.

Acknowledgments: We are grateful to Edoardo Mannelli for having scanned the lateral cephalograms of the Class III patients.

Conflicts of Interest: The authors declare no conflict of interest.

\section{References}

1. Rongo, R.; D'Antò, V.; Bucci, R.; Polito, I.; Martina, R.; Michelotti, A. Skeletal and dental effects of Class III orthopaedic treatment: A systematic review and meta-analysis. J. Oral Rehabil. 2017, 44, 545-562. [CrossRef] [PubMed]

2. Woon, S.C.; Thiruvenkatachari, B. Early orthodontic treatment for Class III malocclusion: A systematic review and meta-analysis. Am. J. Orthod. Dentofac. Orthop. 2017, 151, 28-52. [CrossRef] [PubMed]

3. Cordasco, G.; Matarese, G.; Rustico, L.; Fastuca, S.; Caprioglio, A.; Lindauer, S.J.; Nucera, R. Efficacy of orthopedic treatment with protraction facemask on skeletal Class III malocclusion: A systematic review and meta-analysis. Orthod. Craniofac. Res. 2014, 17, 133-143. [CrossRef]

4. Lin, Y.; Guo, R.; Hou, L.; Fu, Z.; Li, W. Stability of maxillary protraction therapy in children with Class III malocclusion: A systematic review and meta-analysis. Clin. Oral Investig. 2018, 22, 2639-2652. [CrossRef]

5. Yang, X.; Li, C.; Bai, D.; Su, N.; Chen, T.; Xu, Y.; Han, X. Treatment effectiveness of Fränkel function regulator on the Class III malocclusion: A systematic review and meta-analysis. Am. J. Orthod. Dentofac. Orthop. 2014, 146, 143-154. [CrossRef]

6. Garattini, G.; Levrini, L.; Crozzoli, P.; Levrini, A. Skeletal and dental modifications produced by the Bionator III appliance. Am. J. Orthod. Dentofac. Orthop. 1998, 114, 40-44. [CrossRef]

7. Mousoulea, S.; Tsolakis, I.; Ferdianakis, E.; Tsolakis, A.I. The effect of chin-cup therapy in Class III malocclusion: A systematic review. Open Dent. J. 2016, 10, 664-679. [CrossRef]

8. Tollaro, I.; Baccetti, T.; Franchi, L. Mandibular skeletal changes induced by early functional treatment of Class III malocclusion: A superimposition study. Am. J. Orthod. Dentofac. Orthop. 1995, 108, 525-532. [CrossRef]

9. Tollaro, I.; Baccetti, T.; Franchi, L. Craniofacial changes induced by early functional treatment of Class III malocclusion. Am. J. Orthod. Dentofac. Orthop. 1996, 109, 310-318. [CrossRef] 
10. Franchi, L.; Baccetti, T.; Tollaro, I. Predictive variables for the outcome of early functional treatment of Class III malocclusion. Am. J. Orthod. Dentofac. Orthop. 1997, 112, 80-86. [CrossRef]

11. Baccetti, T.; Tollaro, I. A retrospective comparison of functional appliance treatment of Class III malocclusions in the deciduous and mixed dentitions. Eur. J. Orthod. 1998, 20, 309-317. [CrossRef]

12. Saleh, M.; Hajeer, M.Y.; Al-Jundi, A. Assessment of pain and discomfort during early orthodontic treatment of skeletal Class III malocclusion using the Removable Mandibular Retractor Appliance. Eur. J. Paediatr. Dent. 2013, 14, 119-124. [PubMed]

13. Saleh, M.; Hajeer, M.Y.; Al-Jundi, A. Short-term soft- and hard-tissue changes following Class III treatment using a removable mandibular retractor: A randomized controlled trial. Orthod. Craniofac. Res. 2013, 16, 75-86. [CrossRef] [PubMed]

14. Majanni, A.M.; Hajeer, M.Y. The removable mandibular retractor vs the bone-anchored intermaxillary traction in the correction of skeletal Class III malocclusion in children: A randomized controlled trial. J. Contemp. Dent. Pract. 2016, 17, 361-371.

15. Lavergne, J.; Gasson, N. Operational definitions of mandibular morphogenetic and positional rotations. Scand. J. Dent. Res. 1977, 85, 185-192. [CrossRef]

16. Kuc-Michalska, M.; Baccetti, T. Duration of the pubertal peak in skeletal Class I and Class III subjects. Angle Orthod. 2010, 80, 54-57. [CrossRef]

17. McNamara, J.A., Jr.; Franchi, L. The cervical vertebral maturation method: A user's guide. Angle Orthod. 2018, 88, 133-142. [CrossRef] [PubMed]

18. Pavoni, C.; Masucci, C.; Cerroni, S.; Franchi, L.; Cozza, P. Short-term effects produced by rapid maxillary expansion and facemask therapy in Class III patients with different vertical skeletal relationships. Angle Orthod. 2015, 85, 927-933. [CrossRef]

19. Springate, S.D. The effect of sample size and bias on the reliability of estimates of error: A comparative study of Dahlberg's formula. Eur. J. Orthod. 2012, 34, 158-163. [CrossRef] [PubMed]

20. Galeotti, A.; Martina, S.; Viarani, V.; Franchi, L.; Rongo, R.; D'Antò, V.; Festa, P. Cephalometric effects of Pushing Splints 3 compared with rapid maxillary expansion and facemask therapy in Class III malocclusion children: A randomized controlled trial. Eur. J. Orthod. 2021, 43, 274-282. [CrossRef]

21. Fabozzi, F.F.; Nucci, L.; Correra, A.; d’Apuzzo, F.; Franchi, L.; Perillo, L. Comparison of two protocols for early treatment of dentoskeletal Class III malocclusion: Modified SEC III versus RME/FM. Orthod. Craniofac. Res. 2021, 24, 344-350. [CrossRef]

22. Vetlesen Westwood, P.; McNamara, J.A., Jr.; Baccetti, T.; Franchi, L.; Sarver, D.M. Long-term effects of Class III treatment with rapid maxillary expansion and facemask therapy followed by fixed appliances. Am. J. Orthod. Dentofac. Orthop. 2003, 123, 306-320. [CrossRef] [PubMed]

23. Masucci, C.; Franchi, L.; Defraia, E.; Mucedero, M.; Cozza, P.; Baccetti, T. Stability of rapid maxillary expansion and facemask therapy: A long-term controlled study. Am. J. Orthod. Dentofac. Orthop. 2011, 140, 493-500. [CrossRef] 